\title{
Throat Swabs in Primary Care
}

\section{Ton TA, Sheldon A, Young D, Clark LJ, Montgomery J, Douglas CM \\ Department of Otolaryngology, Queen Elizabeth University Hospital, Glasgow}

\section{Introduction}

Throat swabs in Primary Care have limited value in management of sore throat and are not recommended by the Scottish Intercollegiate Guideline Network (SIGN) ${ }^{1}$. Reasons include: asymptomatic carriers of Group A Streptococcus (10-20\%), sensitivity, cost, medicalisation of illness and do not alter immediate management.

The aim of the project was to establish the appropriateness of SIGN guidelines and to determine which members of society received throat swabs and referral to Ear, Nose and Throat specialists.

\section{Methods}

Bacterial throat swabs performed in Primary Care were identified in one year. Demographics, swab results, Scottish Index of Multiple Deprivation (SIMD) and referrals for tonsillectomy were recorded using Community Health Index (CHI) and the online Clinical Portal System.

\section{Results}

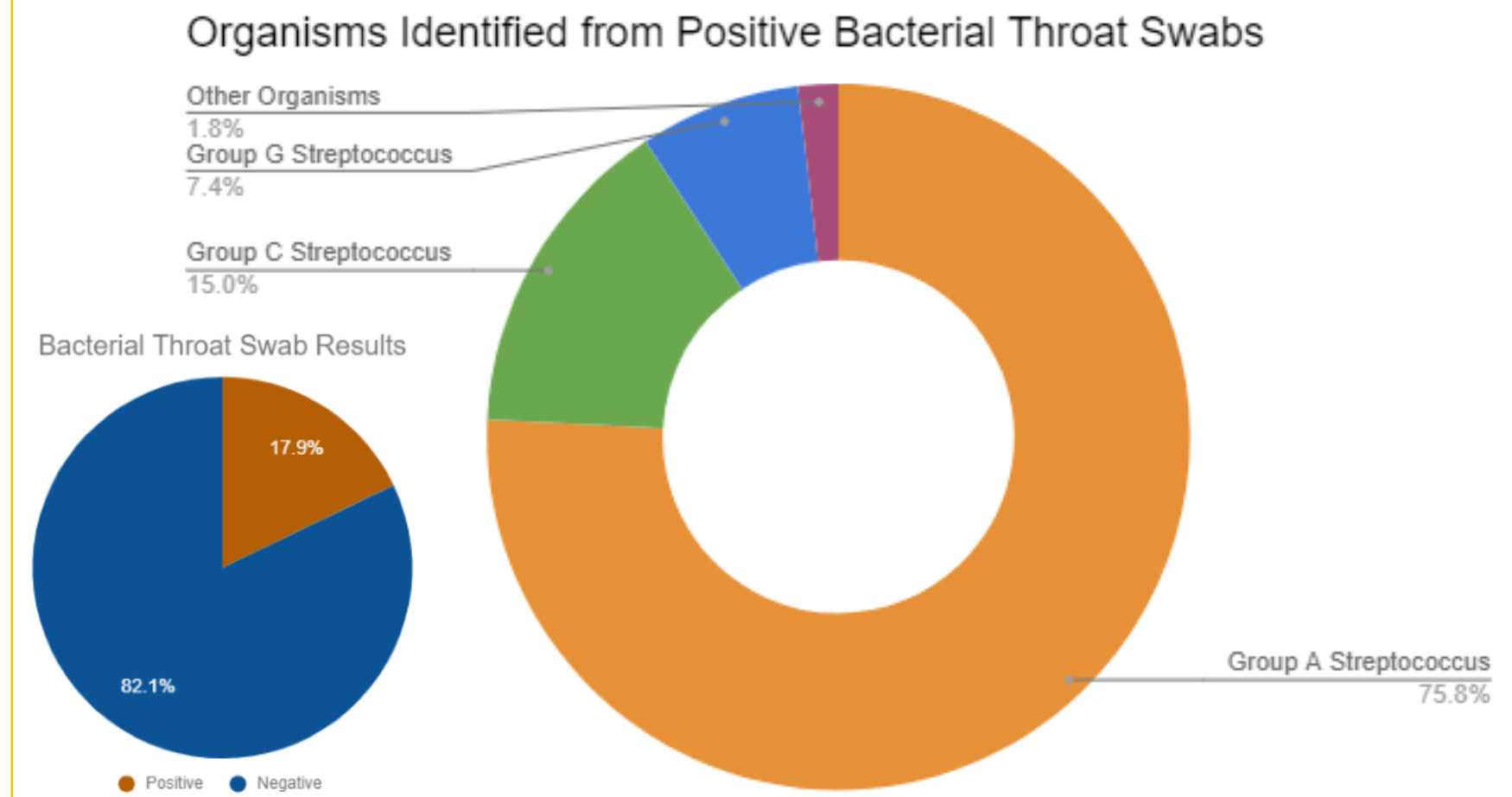

Fig. 1a: (left) The percentage of positive vs negative bacterial throat swab cultures. Fig. 1b: (right) The distribution of organisms identified from positive bacterial throat swab cultures. Other organisms includes - Staphylococcus aureus, Group Between April 2014 and April 2015, 2690 swabs were performed in Glasgow. 487 (18.1\%) were positive. The most common organism was Group A Streptococcus (369; 76\%).

Mean patient age was 28.77. $1658(62.1 \%)$ of swabs were females. Mean SIMD was 3740.81 (Quintile $=3$ )*. $298(11 \%)$ patients were referred to ENT. 106 (3.9\%) patients had tonsillectomy.

For each quintile, a chi goodness of fit test was performed to examine population data from Glasgow, and to look at the number of swabs and referrals in the context of quintile population data. More patients than expected were swabbed $(p<0.001)$, referred to secondary care $(p<0.001)$, and underwent tonsillectomy $(p<0.007)$ in Quintile 5 compared to Quintile 1. * Quintile $1=$ most deprived. Quintile $5=$ least deprived.

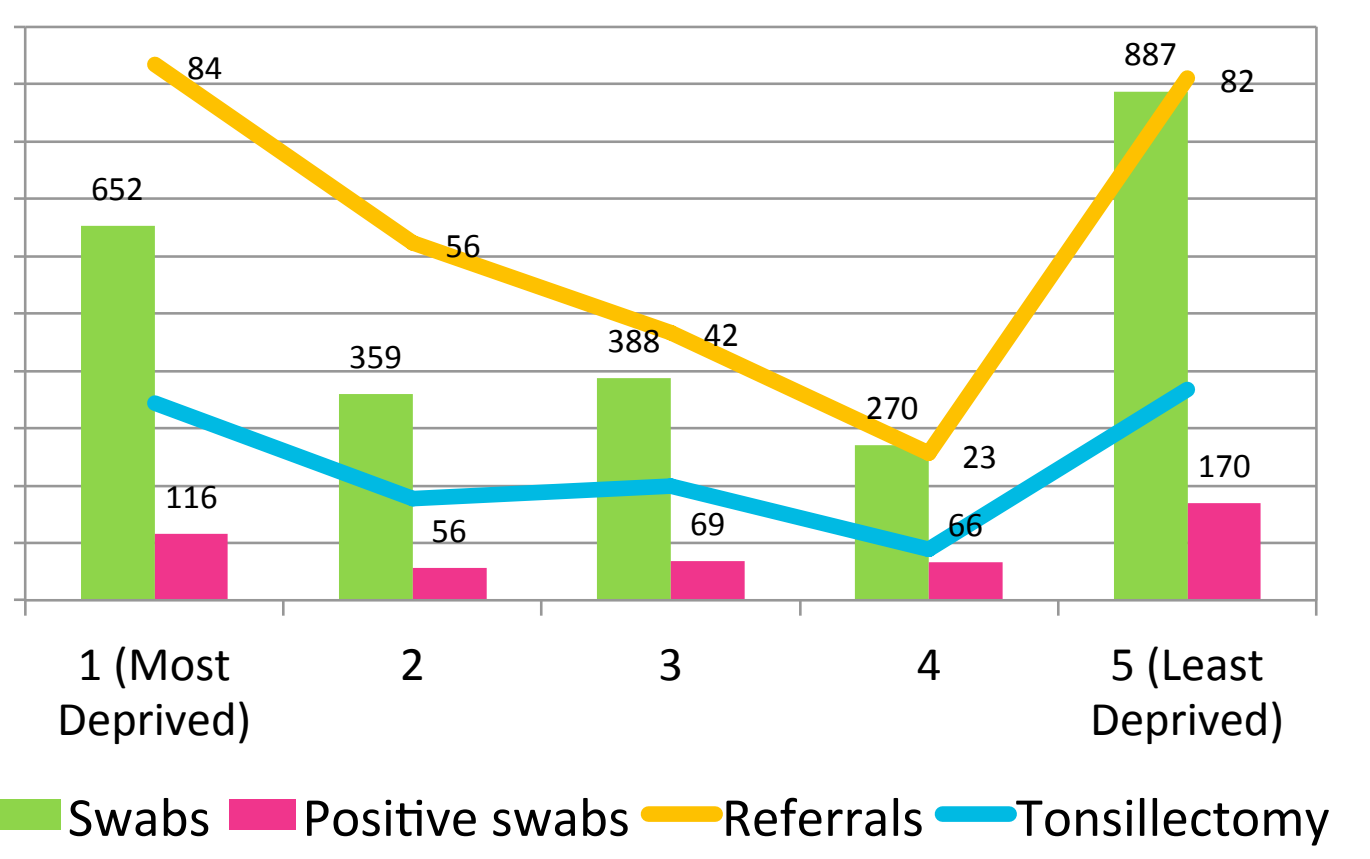

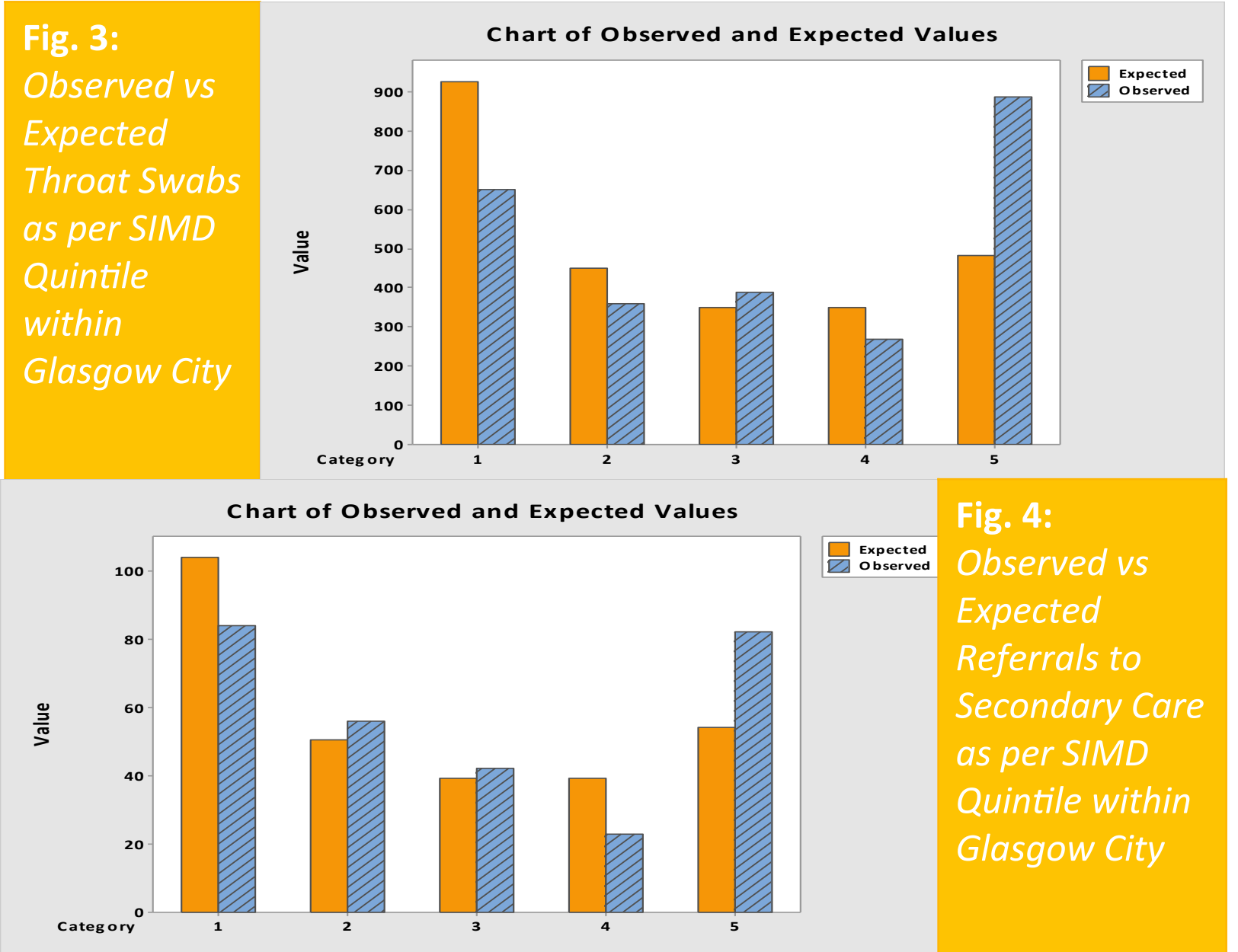

\section{Discussion}

\section{Appropriateness of SIGN Guidelines}

\section{Sensitivity and specificity}

SIGN suggests that routine throat swabs are not sensitive or specific for Group A streptococcal. The results show that throat swabs were positive for growth in only $18.1 \%$ of swabs. This suggests that patients who were being swabbed for sore throat with negative results were likely due to viral infections.

\section{Cost effectiveness}

In the Greater Glasgow and Clyde health board, each bacterial charcoal throat swab costs a total of $£ 25.00$ to use, test and culture. Total cost for one years worth of throat swab in one heath board equated to $f 67,125$ on throat swabs alone.

\section{Do not alter immediate management plans}

Throat swabs take up to three days to grow cultures in which management of sore throat should have been decided. The results show that positive culture also had very little impact on who was referred to secondary care and ultimately received tonsillectomy.

\section{Medicalise illness}

Only a small number of throat swabs were positive showing that the most likely cause of sore throat is secondary to viral illness which is self limiting. Medicalisation of illness may be taken more seriously in patients from higher socioeconomic backgrounds

\section{Deprivation}

This study highlights that deprivation had an impact on which patients received throat swabs and further management. When comparing populations within each deprivation quintile a greater number of patients than expected received throat swabs, referral to secondary care and underwent tonsillectomy within the least deprived quintile. There is a strong link between deprivation with comorbidity which would suggest that it should be the deprived receiving more investigations and referrals to prevent morbidity.

The results may be a consequence of those in higher socioeconomic backgrounds having higher health literacy and may have an expectation for additional investigations, plus GP attitudes to swabbing e.g. to justify antibiotic prescription and maintaining good doctor-patient relationship.

\section{Conclusion}

Deprivation has a significant association to receiving swabs, referral to ENT and tonsillectomy. Swab outcome did not affect further management. The SIGN recommendations against throat swabs are appropriate. 